\title{
Una aproximación entre la psicología positiva y el apoyo conductual positivo
}

\section{An approach between positive psychology and positive behavioral support}

\begin{abstract}
Resumen
La psicología positiva y el apoyo conductual positivo tienen orígenes diferentes; sin embargo, abordan objetivos similares. En este trabajo se describe la psicología positiva y el apoyo conductual positivo, así como algunas visiones críticas. Posteriormente, a través de Carr, un pionero del apoyo conductual positivo, se aborda el acercamiento entre ambos modelos. Por su parte, la psicología positiva está incluyendo a las personas con discapacidad, según dos manuales de psicología positiva y discapacidad; anteriormente, según éstos, las personas con discapacidad no estaban incluidas en el modelo. Por otra parte, también se describen las teorías de dos autores, Wrigth (2005) y Pinker (2012), que explican la evolución de la sociedad actual hacia los valores positivos. En parte, esta evolución explica la buena acogida de las psicologías positivas. Finalmente, la visión contextual y social podría ser la principal aportación del apoyo conductual positivo a la positividad. Los cambios de entornos y las relaciones sociales han sido temas centrales para el apoyo conductual positivo; sin duda, para la psicología positiva también, no obstante, su principal aportación se centra en los factores intrapsíquicos.
\end{abstract}

\section{Palabras clave}

Psicología positiva, apoyo conductual positivo, discapacidad, psicología.

\begin{abstract}
Positive psychology and positive behavioral support have different origins; however, they address similar goals. In this work, positive psychology and PBS are described, as well as some critical views. Subsequently, through Carr, a pioneer of the positive behavioral support, the rapprochement between both models is addressed. For its part, positive psychology is including people with disabilities, according to two positive psychology and disability manuals; previously, according to these, people with disabilities were not included in the model. On the other hand, the theories of two authors, Wright (2005) and Pinker (2012), who explain the evolution of today's society towards positive values are also described. In part, this evolution explains the good reception of positive psychologies. Finally, the contextual and social vision could be the main contribution of the positive behavioral support to positive psychology. Changes in environments and social relationships have been central themes for the positive behavioral support; undoubtedly, for positive psychology as well, however, its main contribution focuses on intrapsychic factors.
\end{abstract}

\section{Keywords}

Positive psychology, positive behavioral support, disability, psychology.

\author{
Sebastià Forteza Bauzá \\ <sebastia.forteza@aprop.org> \\ Consorci Aprop. España

\section{Vicenç Ferretjans Moranta <dicopib@gmail.com>}

Centro Mater Misericordiae. España

\section{Elisabeth Vilardebó \\ Perxachs \\ <beth@aproscom.org>}

Aproscom Fundació. España

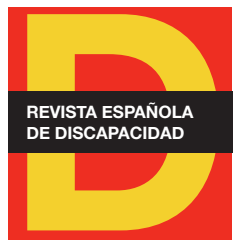

Para citar:

Forteza, S. et al. (2021). Una aproximación entre la psicología positiva y el apoyo conductual positivo. Revista Española de Discapacidad, 9(1), pp. 197-215.

Doi: <https://doi.org/10.5569/23405104.09.01.10>

Fecha de recepción: 26-06-2020 Fecha de aceptación: 14-05-2021

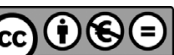




\section{Introducción}

La psicología positiva y el apoyo conductual positivo son dos modelos que investigan y promocionan el bienestar. El primero dirigido a la población general y el segundo a personas con discapacidad y otros colectivos como escuelas ordinarias, centros de acogida juvenil y tercera edad. Ambos modelos comparten principios similares: el bienestar a través de las emociones, pensamientos y prácticas conductuales. Básicamente, las técnicas que usan son las clásicas de la psicología; por ejemplo, cambios en la forma de pensar, control emocional, control estimular, técnicas comunicativas, equivalencias funcionales, etcétera (por ejemplo, véase Berrocal et al. 2008; Carr et al., 1999; Chaves et al., 2016; Fernández-Abascal, 2009; Lavigna et al., 2009; Park et al., 2013. En la actualidad, a estas estrategias se suman constructos generales como felicidad, perdón, optimismo, resiliencia, calidad de vida o autodeterminación. Al comentar la positividad habría dos niveles: la perspectiva empírica y la del modelo general, es decir, su filosofía.

La positividad se presenta como una nueva visión de la psicología; esta calificación enmascara, tal vez, algunos antecedentes que explican su excelente y popular acogida. Posiblemente, lo que se ha producido es un lento cambio de valores con relación al bienestar. Por tanto, indagando este cambio se podrían añadir puntos de vista a la reflexión positiva. En definitiva, estos modelos surgen dentro de la civilización empática, calificativo que utilizó Rifkin (2010) para referirse al sentir actual.

Por otra parte, la positividad tiene detractores que abren un interesante debate sobre el impacto de estos modelos. Son reflexiones a considerar, en tanto en cuanto la duda es un aliciente científico. Quienes critican sugieren interrogantes sobre la capacidad de los constructos usados porque son muy generales y poco operativos; más allá, también critican su visión crédula, poco realista y conformista con ciertos entornos (Pérez-Álvarez et al., 2018; Piña, 2015).

En cualquier caso, son modelos populares (Yakushko y Blodgett, 2018). Su huella trasciende lo exclusivamente empírico al promocionar un estilo de vida y unos valores. Esto explica, al menos en parte, su influencia sobre clínicos, profesionales y público general, quienes toman el modelo positivo en su conjunto. Como subraya Taleb (2016, p. 433), existe "una tendencia innata a sentir debilidad por las teorías. Pero las teorías van y vienen; lo que permanece es la experiencia. Las explicaciones cambian continuamente...la experiencia, por su parte, se mantiene constante".

Por todo ello, en este trabajo se describirán características de ambos modelos y algunas críticas. Seguidamente, se comentarán contactos y cruces entre la psicología positiva y el apoyo conductual positivo; Carr (2007) señalaba que la psicología positiva es el aliado más potente del apoyo conductual positivo. A continuación, se resaltarán dos visiones de cómo han cambiado los valores actuales. Por último, se subrayará que el deseo de cambiar los contextos negativos y las relaciones humanas son, tal vez, los valores más importantes que el apoyo conductual positivo aporta a la positividad, es decir, para el apoyo conductual positivo cambiar contextos y situaciones es una tesis básica y la interacción humana podría ser el núcleo de esa tesis. Este trabajo pretende seguir una perspectiva reflexiva y abierta. 


\section{La psicología positiva}

Linley et al. (2006) definen la psicología positiva de diez maneras diferentes, que se podrían sintetizar en el estudio y aplicación de los valores psicológicos dirigidos al bienestar, optimismo, esperanza, felicidad y fluidez. La intervención se desarrolla en grupo o individualmente. Según estos autores, la psicología positiva trabaja en dos líneas: la metapsicológica y la pragmática. La primera define, clasifica e integra los constructos (es decir, la psicología positiva como modelo). La segunda investiga las técnicas y los procedimientos concretos.

La psicología positiva inicia su andadura en 1998 cuando Martín Seligman pasó a presidir la American Psychological Association. La psicología precisaba un cambio porque se había centrado excesivamente en la enfermedad, es decir, en la terapia y en las emociones negativas (ansiedad, depresión, estrés). En definitiva, se estaba frente a una psicología reactiva. La contrapartida era crear una nueva visión centrada en lo positivo, por tanto, preventiva. La premisa fue trabajar sobre las fortalezas del ser humano para llegar a una vida plena (Lupano y Castro, 2010). Los autores positivos sitúan a la psicología positiva como la "quinta fuerza" de la psicología, en consecuencia, la consideran al nivel del psicoanálisis o del conductismo, entre otras escuelas (Yakushko y Blodgett, 2018).

Por su parte, los estudios demuestran la relevancia de los constructos positivos. Por ejemplo, un metaanálisis de LyubomirsKy et al. (2005) analiza 225 trabajos, con una muestra de 275.000 personas, y un amplio rango de variables (relaciones humanas, salud, éxito, percepción social, creatividad, altruismo, etcétera). Por citar dos: el bienestar influye en el altruismo y en el sistema inmunitario. A pesar de todo, el trabajo indica algunos puntos críticos. Así, se halla un rendimiento más pobre cuando las tareas son complejas; en otras palabras, podría ser que el bienestar empeorara los resultados. Por otra parte, las autoras dejan abierta la posibilidad de terceras variables que pudieran explicar estos resultados; en concreto, podría ser que la capacidad cognitiva (y no el bienestar) diera cuenta tanto de la felicidad como del rendimiento óptimo.

Por último, McNulty y Fincham (2012) matizan algunos resultados de la psicología positiva; consideran que, tal vez, se sobregeneralizan. Concretamente, si se introducen contextos específicos, se observa que los resultados cambian. Por ejemplo, el perdón (un tópico de la positividad) es saludable para el buen funcionamiento del matrimonio; no obstante, al introducir aspectos como la hostilidad de la pareja, el perdón cambia de valencia y pasa a influir negativamente. Análisis similares se hallan con el optimismo y la amabilidad. Resultados como estos, que cambian sus consecuencias al cambiar ciertos valores, señalan que las conclusiones puede que no sean lineales. Taleb (2016) ha disertado ampliamente sobre el error de las ciencias al ofrecer conclusiones lineales frente a realidades que pudieran representarse de forma cóncava o convexa (esto significa que puede haber puntos críticos donde un resultado cambia de positivo a negativo o viceversa). En ocasiones, por falta de suficientes datos no se captan los cambios. Precisamente, un debate no menor que hay entre defensores y detractores es que mucha felicidad podría acabar produciendo infelicidad.

En definitiva, parece que la psicología positiva actúa con unos límites amplios y, tal vez, difusos (con unas diez definiciones posibles como indican Linley et al., 2006); esto, lógicamente, obliga a sintetizar y a elegir términos o indicadores que den cuenta del modelo; esto es, obliga a definir adecuadamente qué es y no es la psicología positiva. Por su parte, los estudios parecen avalar la importancia de centrarse en lo positivo; no obstante, como señalan estos mismos estudios, son conclusiones que deben considerarse abiertas porque no son definitivas (LyubomirsKy et al., 2005). 


\section{Críticas a la psicología positiva}

Cuando se crea una corriente - parece inevitable - surgen los defensores y los detractores que, en el caso de la psicología positiva, han llegado a calificarse de amigos y enemigos (son términos usados en algunos trabajos publicados en la revista Papeles del Psicólogo; en concreto, Pérez-Álvarez, 2013; Vázquez, 2013). Estas posiciones, en ocasiones acaloradas, pueden sesgar las valoraciones sobre la teoría y la interpretación de los datos, que acaban relativizándose sin posibilidad de encuentro. Por ejemplo, para los defensores la psicología positiva es una novedad (Vera, 2006), que ha dado "un impulso al estudio de lo positivo, que hasta hace pocos años era muy escaso” (Vázquez y Hervás, 2008, p. 23). Para los críticos son conocimientos antiguos de temas tratados (Fernández-Ríos y Novo, 2012) o similares a los de la psicología humanista (Yakushko y Blodgett, 2018).

Los críticos también dudan del verdadero impacto de lo positivo, sugieren que desear ser muy positivo acaba siendo negativo (Pérez-Álvarez, 2012). Taleb (2016) defiende que el malestar, al menos en cierta medida, permite apreciar el bienestar (según comenta, el estrés genera fortalezas y la exposición a dosis adecuadas de sustancias malignas nos hace inmunes). Existen críticas más radicales, como la del filósofo Han (2016, p. 12), para quien: "la violencia de la positividad no es privativa, sino saturativa; no es exclusiva, sino exhaustiva". Los defensores, por su parte, contestan que: "nadie afirma, al menos en la arena académica, que esos elementos positivos sean ilimitadamente beneficiosos en cualquier circunstancia” (Vázquez, 2013, p. 93).

Por otra parte, algunos creen innecesario crear un nuevo modelo (ya se ha comentado, la psicología positiva pasaría por ser psicología humanista) o piensan que los constructos que utilizan son generales y poco operativos (Piña, 2014). Este mismo autor, Piña (2015), analiza la resiliencia y halla inconcreción, ya que, según parece, se utiliza indistintamente como un proceso, un resultado o un atributo de la persona. Posiblemente, esta crítica podría generalizarse a otros conceptos positivos.

También se critica el excesivo y llamativo lenguaje utilizado al difundir los conocimientos. Por ejemplo, al proclamar que "ha llegado el momento de involucrarnos cada vez más, desde diferentes ámbitos de aplicación (sean educativos, clínicos, empresariales, comunitarios, etc.), en intentar promover el bienestar de la ciudadanía" (Vázquez y Hervás, 2009, p. 16). Este lenguaje parece dirigirse al ánimo del lector, porque, al fin y al cabo, se trata de una afirmación incuestionable e incontestable.

Tampoco es de extrañar que tanto los defensores como los críticos vayan más allá del cometido psicológico. Por ejemplo, hay autores que se preguntan si la psicología positiva manipula para crear conformismo con algunos sistemas establecidos (Han, 2016; Pérez-Álvarez et al., 2018). El motivo es que, al hacer recaer el peso del bienestar en las capacidades de la persona, el contexto pasa a un segundo plano; es decir, las situaciones no son cuestionadas. Según los críticos, la psicología positiva es una teoría que pivota principalmente en la autogestión (Pérez-Álvarez, 2012). Si es así, los críticos creen que los entornos perjudiciales tienen una coartada perfecta porque todo el malestar provocado se debe a la debilidad del sujeto. Cuando quien sufre malestar insiste en su crítica es etiquetado de pesimista o derrotista (Pérez-Álvarez et al., 2018). Han (2016, p. 14) también cree que: "la positividad del poder es mucho más eficiente que la negatividad del deber". De esta manera la organización, el sistema, la empresa o el partido no ponen en duda sus posibles presiones o injusticias. Lógicamente, los defensores piensan lo contrario: la psicología positiva no es una "siniestra aliada del individualismo y el capitalismo", es más "hay pocas áreas de investigación en el ámbito 
de las ciencias sociales que, como la psicología positiva, hayan puesto de manifiesto algunas contradicciones internas del sistema económico actual" (Vázquez, 2013, p. 96).

En definitiva, ¿se trata de nuevos conocimientos o ya había líneas abiertas anteriormente? Las dudas se extienden a los grados de bienestar, es decir, ¿cuándo mucho bienestar es perjudicial, si lo es en algún nivel? El uso de términos genéricos y amplios (como resiliencia) es la siguiente cuestión que unos y otros observan de forma diferente. Por último, ¿es la positividad, como modelo, una forma de complicidad con algunas situaciones indebidas o, todo lo contrario, es un modelo que cambia situaciones injustas?

\section{El apoyo conductual positivo}

En menor medida, el apoyo conductual positivo también presenta sus definiciones con límites amplios. Posiblemente, esta menor medida se debe al uso del análisis conductual aplicado que unifica y abrazan todos los autores. Carr (2007) añade al análisis conductual la calidad de vida (además de felicidad y optimismo). Para Manokara, et al. (2018) se trata de una corriente de análisis conductual aplicado, modificado con los valores actuales que tienen los servicios. En Johnston et al. (2006) se describe la diversidad de aproximaciones y constructos, centrados todos ellos en el énfasis de los valores. Por otra parte, según explican Johnston et al. (2006, p. 54) "estos valores son bien conocidos en el campo de las discapacidades del desarrollo y durante mucho tiempo han sido aceptadas por diversas profesiones al servicio de esta población, incluida la ABA" (ABA acrónimo en inglés de la asociación de Análisis Conductual Aplicado). Quizá, como aseveran algunos, Kincaid (2018) por ejemplo, mantener una visión abierta es una fortaleza.

En cualquier caso, existe un motivo incuestionable: el apoyo conductual positivo nació como una reacción a las prácticas aversivas que sufrían las personas con discapacidad intelectual y conductas problemáticas (Carr et al., 1999; Horner et al., 1990). Su buena acogida permite que se haya difundido a escuelas ordinarias, servicios de tercera edad y protección juvenil. Su cometido es llegar a los servicios y profesionales para que los usuarios disfruten de una vida similar a la de cualquier otro ciudadano (Horner et al., 1990; Johnston et al., 2006).

Si se observa la evolución del apoyo conductual positivo, se obtiene una mejor perspectiva. Al inicio de la modificación del comportamiento era habitual, frente a las conductas problemáticas, el uso del castigo de forma indiscriminada. Esta desagradable situación cambió gracias a quienes creían que lo punitivo no era ético (Horner et al., 1990; Johnston et al., 2006; Jones y McCaughey, 1992). Por eso, paso a paso, surgió un movimiento a favor del uso exclusivo de "practicas positivas". Es decir, la primera acepción de "positivo" iba en contra del castigo y en su lugar se aconsejaba el uso exclusivo de técnicas positivas, como los refuerzos diferenciales, las equivalencias funcionales, el control del estímulo, los programas de enseñanza de habilidades y de comunicación (Horner et al., 1990). Existen, cabe acentuarlo, antecedentes del apoyo conductual positivo: a mediados de los 80 un grupo de psicólogos de la universidad de Nebraska ya abominaron del castigo, esta corriente fue denominada "enseñanza suave" (Jones y McCaughey, 1992).

Por su parte, el término conductual junto al término positivo reivindicaba los procedimientos del análisis conductual aplicado, el cual contaba con una perspectiva empírica y operativa que garantizaba un modelo 
de trabajo científico. Por último, los apoyos remiten al paradigma que posteriormente recogió la definición sobre la discapacidad intelectual.

Hoy el término positivo ha cambiado, va más allá de las técnicas. Ahora señala, igual que la psicología positiva, una promoción de las fortalezas y capacidades (Carr, 2007). El apoyo conductual positivo también trabaja e incorpora, además del análisis conductual, los constructos que han ido apareciendo en el mundo de la discapacidad; por ejemplo, calidad de vida, autodeterminación, participación, inclusión, etcétera. En definitiva, se habla de cambiar no sólo al usuario sino su estilo de vida y su entorno. Horner y Sugai (2018) describen esta doble vertiente como el matrimonio de los valores con la ciencia.

Como se ha indicado, su impacto está fuera de toda duda. Por ejemplo, es suficiente prueba que unas 25.000 escuelas de EEUU lo hayan asumido (Pinkelman y Horner, 2019). Según Johnston et al. (2006), existe un consorcio de universidades creado durante el quinquenio 87-92 con centros de rehabilitación, investigación y entrenamiento (RRTC) que hoy se denomina RRTC-PBS (PBS son las siglas en inglés de apoyo conductual positivo). Por otra parte, existen revistas (por ejemplo, Journal of Positive Behavior Interventions; International Journal of Positive Behavioural Support) y asociaciones (por ejemplo, Association for Positive Behavior Support, APBS).

\section{Críticas al apoyo conductual positivo}

Las críticas son similares a las de la psicología positiva pero más escasas, tal vez, porque su ámbito de aplicación, la población a la que se dirige, es más vulnerable. Los críticos dejan claro que no atacan la positividad ni van en contra de los principios éticos que sustentan el deseo de maximizarla. Tampoco defienden el castigo, en sí mismo. Al contrario, lo que realmente desean es manifestar su desacuerdo con los constructos utilizados porque carecen de suficiente validez y capacidad operativa, en resumen, los términos positivos son genéricos y poco discriminativos (Foxx, 2005; Mulick y Butter, 2005; Newson y Kroeger, 2005). Por ejemplo, para Foxx (2005) el apoyo conductual positivo es un mito que ejerce un efecto narcótico en las jóvenes generaciones más atraídas por el discurso positivo que por su validez empírica. Este autor considera que el apoyo conductual positivo no brega con conductas realmente complejas; conductas como, por ejemplo, las que describen Spicer y Crates (2016) autores positivos que deben afrontar situaciones duras y complejas (agresiones, destrucción, amenazas, etcétera). Por otra parte, Mulick y Butter (2005) tildan al apoyo conductual positivo de "ilusión paternalista utópica", creen que la positividad llega hasta el análisis conductual aplicado y a partir de ahí el resto es ideología; la palabra "positivo", dirán, ejerce un efecto ilusorio. Newson y Kroeger (2005) hallan un discurso plagado de conceptos sin claras definiciones y fronteras; por ejemplo, aversivo y abusivo se usan indistintamente, lo mismo que dolor, humillación y deshumanización al referirse a todas las técnicas aversivas (por otra parte, creen que es un error comentar las técnicas aversivas como si todas fueran iguales).

Los críticos piensan, por otra parte, que el apoyo conductual positivo es endogámico: los autores se citan entre sí, refieren pocos datos y dan por supuesto incuestionable sus principios. En ocasiones, cuando no se obtienen suficientes éxitos, esta endogamia se encierra en una burbuja y achaca los fracasos a la falta de 
implicación de los profesionales o los servicios con los principios del apoyo conductual positivo (Johnston et al., 2006; Mulick y Butter, 2005).

Finalmente, también se cuestiona si la positividad del apoyo conductual positivo pudiera ser, de alguna forma y en algún caso, negativo. Por ejemplo, se han descrito situaciones conflictivas que podrían solucionarse con una recompensa; es decir, alguien que está manifestando conductas negativas recibe, para que deje de realizarlas, algún estímulo positivo (esta estrategia se conoce como capitulación). En el debate hay quienes estarían a favor y quienes en contra. Los primeros creen que las situaciones críticas deben detenerse aun a costa de un estímulo preferente (por supuesto, también creen que los programas proactivos diseñados adecuadamente acabarán con esos conflictos; por tanto, la capitulación es temporal, puntual y estratégica). Otros ven ilógico solucionar una conducta desdeñable dando un estímulo preferente que podría reforzar (y desear repetir) el conflicto. Spicer y Crates (2016), defienden y ofrecen algunos datos a favor de la postura apoyo conductual positivo. Por otra parte, y que nos conste, apenas existe bibliografía con referencia a las estrategias reactivas no aversivas.

\section{Encuentros entre el apoyo conductual positivo y la psicología positiva}

Para Carr (2007) la psicología positiva y el apoyo conductual positivo tratan tres principales temas: felicidad, amabilidad y optimismo. Este autor inició una aproximación entre ambas corrientes; que su trágico fallecimiento (en 2009) posiblemente frenó. Este autor describió que:

"La amplitud, profundidad y variedad que caracteriza a la psicología positiva y su evidente vinculación conceptual y práctica al apoyo conductual positivo es una fuente de esperanza para nuestro campo y deja claro que no somos una voz solitaria en el desierto: somos una de las muchas voces de un coro" (Carr, 2007, p. 11).

Es interesante comprobar que su expectativa iba más allá de lo conceptual, veía una vinculación práctica. Vinculación, se podría interpretar, como un uso común de estrategias porque sus principios e impacto sobre las personas son comunes; en otras palabras, subrayar lo práctico es un paso que va más allá de la pura visión teórica o pura manifestación formal.

Años antes, este autor señaló un hecho relevante con respecto a la igualdad de las personas con y sin discapacidad al adquirir ciertas competencias positivas, hecho no siempre apreciado con suficiente credibilidad:

"Los intereses compartidos constituyen la base de la simpatía y de la amistad entre las personas que no tienen discapacidad, y no hay razón para que no deban ser también la base para el desarrollo de la simpatía y la amistad, para las personas discapacitadas" (Carr et al., 1996, p. 143).

Esta cita, en nada gratuita, señalaba que había quienes creían que las personas con discapacidad se regían por principios mentales diferentes a los principios que rigen la mente de las personas ordinarias. Esta visión sobre igualdad es un importante motor del trato positivo. Si se observa lo contrario, se evidencia de forma clara. El trato inhumano se rige por la creencia de principios mentales diferentes (en la desigualdad histórica entre el hombre y la mujer, por ejemplo, se hallan descripciones de las diferencias psicológicas; véase, por ejemplo, Rifkin, 2010). 
Por su parte, desde la psicología positiva se observa que las personas con discapacidad comienzan a ser objeto de su interés. Es interesante observarlo en dos recientes manuales que tratan psicología positiva y discapacidad, sin mencionar el apoyo conductual positivo. El primero, lo edita Wehmeyer (2013b) y, el segundo, Shogren et al. (2017). En síntesis, según comenta Wehmeyer (2013a), la psicología positiva no se aplicaba a poblaciones que tuvieran enfermedades, y la discapacidad, vista exclusivamente como enfermedad, generaba un prejuicio: calificaba a toda la persona de enferma. La definición de discapacidad del año 1992 matizó que no existían discapacitados sino personas con discapacidad (la persona prevalece a cualquier consideración o característica que tenga o padezca, incluidas las enfermedades si las hubiera). En definitiva, despojando a la persona con discapacidad de esa etiqueta que lo definía totalmente, se comenzó a describir la diversidad y, en todo caso, sus características (por ejemplo, simpático, alegre o buena persona, con el mismo rango que cualquier otra descripción incluida la propia deficiencia). Así pues, ahora las personas con discapacidad son un grupo de interés para la psicología positiva.

También se observa, en otra línea (en un trabajo de fin de master), la relación entre la psicología positiva y la discapacidad intelectual en el aprendizaje de habilidades (de la Cal, 2014), sin referirse al apoyo conductual positivo. Si esto se convierte en tendencia, se reforzaría el papel de la psicología positiva con relación a nuestra población.

De cualquier forma, como indicaba el profesor Carr, existe una partitura positiva y el apoyo conductual positivo es uno de los miembros del coro. Por tanto, el autor estaba universalizando la positividad desde un punto de vista amplio. La base de este juicio es que el bienestar es el mismo para todos porque las estrategias y su impacto son idénticos; en otras palabras, todos sentimos y respondemos con las mismas leyes psíquicas, no hay diferencias. Esto significa que si la psicología positiva (o en su caso, el apoyo conductual positivo) hallan nuevos conocimientos o fórmulas de bienestar deben difundirse a todos tengan o no discapacidad. En definitiva, en el futuro, si los lazos y el intercambio de conocimientos son fluidos y próximos, posiblemente, bajo la premisa de conocimientos universales, habrá una ciencia de la positividad y tanto la psicología positiva como el apoyo conductual positivo serán dos patas de esa ciencia.

La investigación de la psicología positiva ha pivotado, no obstante, hacia el estudio de variables intrapsíquicas (felicidad, perdón, optimismo, fortaleza, fluir). Ciertamente, tienden a su aplicación comunitaria (Noble y McGrath, 2015; Seligman et al., 2009). A pesar de ello, aun así, son aplicaciones encaminadas hacia la persona, hacia variables psicológicas. Por ejemplo, el trabajo de Seligman et al. (2009) involucra a los alumnos, maestros y familiares de todo el colegio; no obstante, usan estrategias dirigidas a los pensamientos y emociones (por ejemplo, los alumnos debían escribir tres cosas buenas que les habían pasado). Es decir, se daban estrategias y formación a todas las partes para que comprendieran la sinergia de estas variables (cognitivas) en el rendimiento escolar y en la prevención de conductas. En resumen, en estos estudios el contexto se refiere a la aplicación de estrategias sobre la persona, pero trabajándolo en un colegio. Se obvia el término contexto en un sentido estructural; el trabajo de Seligman et al. (2009), por ejemplo, no describe nada al respecto (ratios de profesores, estructuras de las aulas, gestión del tiempo, etcétera). Esta acepción de estrategias contextuales en términos de recursos o en términos generales es importante para separar claramente unas variables de otras.

Por su parte, el apoyo conductual positivo podría aportar a la positividad una visión de contexto en sentido estructural. El cambio de los contextos para el apoyo conductual positivo ha sido una necesidad. Se comprueba históricamente con servicios alejados de la ciudad, instituciones grandes, formación de los profesionales deficitaria, etcétera. Como se ha indicado, la primera modificación de conducta se centró en 
el cambio de las respuestas del usuario en un entorno empobrecido. Esta incongruencia, se vio pronto, era un sinsentido modificar conductas sin modificar el contexto, tanto social como estructuralmente.

Así pues, con independencia del programa personal o grupal dirigido directamente a las personas, existen modificaciones estructurales o dirigidas al bienestar de forma indirecta. Por ejemplo, en el servicio de uno de los autores se está llevando a cabo la reforma de las habitaciones para que sean individuales en lugar de compartidas (se intuye que esto impactará sobre diversas áreas del bienestar de los usuarios); hace poco, el IMAS (Instituto Mallorquín de Asuntos Sociales) aprobó ayudas para profesionales que apoyarán a las personas que precisarán una atención más individualizada. En resumen, con independencia del término usado, las variables para lograr el bienestar se pueden dirigir a cambiar las habilidades de la persona (variables cognitivas, emocionales y sociales) o a la modificación de su situación y contexto.

Para el apoyo conductual positivo es lógico que algunos modelos de calidad de vida (dirigidos a las personas con discapacidad) tengan un parte objetiva, referida a las condiciones de vida, con una subjetiva, referida a la persona (véase, por ejemplo, Felce y Perry, 1995). Topan con esta diferencia Hassiotis et al. (2018) tras aplicar programas con apoyo conductual positivo sin los éxitos que esperaban; estos autores concluyen que los programas serían óptimos si se añadieran recursos, tal como terapeutas acreditados. En definitiva, para el apoyo conductual positivo el contexto significa cambios en las estructuras arquitectónicas, sociales y de condiciones (además de, o junto a, los programas dirigidos a las personas). Es un hecho, si se observa, que términos como normalización, inserción, participación son de uso habitual en el apoyo conductual positivo, mientras que optimismo, fluir, perdón, agradecer lo son de la psicología positiva. Por supuesto se trata de tendencias, si bien todas las personas reconocen, es obvio, que todo influye en todo.

Para finalizar, cabe resaltar otro factor del contexto: las relaciones humanas entendidas como intercambio para fines comunes. Se quiera o no, todo lo que sucede acaba siendo relación humana específica. Por ejemplo, un maestro que enseña, un profesional que apoya o un tutor que ayuda. En el pasado este rol de alguien que apoya, enseña o guía se ejercía de manera asimétrica; un miembro mantenía el poder sobre la relación, mientras el otro debía mostrarse receptivo. En Poplin (1991) se halla un excelente y clásico trabajo de la asimetría de las relaciones en los antiguos modelos (médico, conductista, cognitivista). La visión que ofrece la autora en la atribución del fracaso, por ejemplo, es que la culpa siempre recaía en el que aprendía (o, en el modelo médico, en el enfermo que debía ser curado) nunca en el que enseñaba; el alumno o la persona con discapacidad era el débil, el profesor, quien tenía el conocimiento y las herramientas para enseñar, nunca era cuestionado. El fracaso, en cualquier caso, se debía a una valoración demasiado benévola; si no aprendía es que las capacidades o habilidades del alumno eran menores que las evaluadas inicialmente. En definitiva, Poplin dibujaba una espiral donde siempre la responsabilidad del fracaso caía del lado débil, quien debía adaptarse o, en caso contrario, debía cambiar de servicio, aula o incluso salir del sistema.

Esta asimetría no tiene ningún papel en el seno de la positividad, donde el buen trato y la corresponsabilidad es esencial. La positividad exige nuevas formas de relacionarse. Ahora los profesionales deben ser sensibles y afectuosos. Para las personas con discapacidad que viven en residencias u hogares, es decir, que reciben servicio íntegro las 24 horas del día, esta positividad adquiere una trascendencia indiscutible. De todas las variables del contexto las interacciones podrían considerarse el núcleo del bienestar (las malas relaciones pasan por ser uno de los peores estresores humanos).

En las relaciones humanas habría, al contrario que en otros constructos positivos, concreción. Es decir, frecuencia de interacciones, número de contactos o la red de amistades; en definitiva, es un constructo operati- 
vo, perceptible y contable. Por otra parte, existen sólidas pruebas empíricas (más allá del sentido común) que confirman el impacto de las relaciones humanas. Por ejemplo, Holt-Lunstad et al. (2010) demuestran con un metaánalisis que las relaciones humanas son tan importantes para la salud y la supervivencia como eliminar el tabaco, y más que hacer deporte o la dieta. Del Cerro et al. (2010), en una población de ratas de laboratorio con daños cerebrales y desórdenes humorales, observaron que las criadas con afecto, cuando fueron adultas mantuvieron conductas maternas, las criadas sin afecto no (es decir, la crianza había modificado la conducta sin modificar las lesiones, que eran idénticas en ambos grupos). Boothby et al. (2017), valorando la belleza y la autenticidad de unas fotos, demuestran que son más bonitas y reales si se ven junto a alguien, que estando solos, y más aún si ese alguien era un conocido. Van Boven y Gilovich (2003) demuestran que la gente es más feliz cuando gasta dinero con otros (comiendo, por ejemplo) que cuando adquiere objetos deseados.

\section{La positividad: desarrollo de una época}

Wrigth (2005) y Pinker (2012) son dos autores que describen la evolución psico-social del ser humano; ambos creen que la sociedad ha cambiado para bien. También piensan que la positividad es una tendencia que va expandiéndose e impactando sobre todos los miembros. Hoy tenemos suficientes ejemplos que demuestran un profundo cambio: igualdad de género, libertad sexual, protección de los menores, educación universal, bienestar animal, etcétera. Por tanto, los valores humanos observados como una evolución histórica matizan el papel innovador de la psicología positiva y del apoyo conductual positivo; de antecedentes pasan a ser consecuentes.

En cualquier caso, lo anterior ni resta ni pone en duda el importante trabajo de estos modelos, en todo caso solo matiza ciertos aspectos. Verdugo y Schalock (2006) defienden la necesidad de modelos porque el progreso científico, médico y tecnológico no ha sido suficiente para mejorar la calidad de vida de las personas con discapacidad. En otras palabras, el bienestar precisa ser activado para que se provoquen o se aceleren los beneficios sociales sobre poblaciones menos favorecidas. Por eso, en otro escrito explican su modelo de calidad de vida como una noción sensibilizadora, guía y marco conceptual de referencia del bienestar (Schalock y Verdugo, 2006). Algún autor crítico con el "optimismo científico" de la psicología positiva creen que difundir el bienestar es importante y necesario (Pérez-Álvarez et al., 2018).

Si la psicología recoge valores sociales que influyen en los modelos debe explicar también cómo surgen y se refuerzan esos valores. Wrigth (2005) para explicarlo se vale de la teoría de juegos. En concreto, los juegos denominados de suma no nula donde los participantes no ganan porque los demás pierden (al contrario que en los juegos de suma cero). La teoría de juegos es un modelo matemático sobre toma de decisiones inciertas, mediante simulaciones de problemas sociales. Entre las simulaciones más conocidas destaca el dilema del prisionero, en el que dos personas tienen la posibilidad de colaborar o no entre sí. Los resultados para cada uno dependen de la respuesta de ambos. Por ejemplo, si al emitir la respuesta uno no colabora y el otro sí, el primero sale beneficiado y el segundo perjudicado; ambos se benefician si colaboran; y ambos salen perjudicados si se defraudan mutuamente. Estos juegos adquieren sentido al ir repitiendo las jugadas, de forma que los participantes se van conociendo entre sí. Los juegos son simulaciones de la realidad; los más críticos con esta teoría resaltan la simulación (creen que esta teoría no dibuja la realidad), los defensores creen que refleja la dinámica real. 
Para Wright, la teoría de juegos es el auténtico motor de la vida y de la cultura sobre la tierra (este autor compara la teoría de juegos para las ciencias sociales, con el ADN para la biología). La llegada al equilibrio tras muchas repeticiones, es decir, tras muchas interacciones, demuestra la existencia de decisiones óptimas que acaban dominando. Wright cree que el mejor resultado tras largas interacciones es la colaboración. En otras palabras, la colaboración entre personas se establece como la única forma social de avanzar. A partir de ahí, que vayan surgiendo - reforzándose - sentimientos positivos aparece como un paso lógico.

El hecho, además, es que existe "la tendencia innata a vigilar las aportaciones de los demás, consciente o inconscientemente. En todas las culturas hay tensiones ocultas en las amistades...sobre quién es un vago y quién es miembro del equipo" (Wrigth, 2005, p. 39). En esta cita se observa la presión social sobre la conducta. Esto obliga y cambia los comportamientos que pueden y desean hacer las personas. Hay evidencias, mediante otro juego denominado el juego del ultimátum, de la tendencia humana hacia la equidad, aunque esa tendencia represente un autosacrificio. En concreto, en este juego el experimentador da 10 euros a un participante para que los reparta con otro de la forma que quiera y éste otro puede aprobar o rechazar. Si aprueba, cada jugador se queda con su parte y si rechaza, ambos lo pierden todo. Si el primer jugador reparte de forma poco equitativa (por ejemplo, se queda 8 y reparte 2) el segundo suele rechazarlo. Este juego demuestra que se prefiere perder (sacrificar un beneficio, aunque sea pequeño) con tal de castigar al injusto, quien desea quedarse con un trozo de tarta excesivamente grande. En resumen, el intercambio social acaba en cooperación y, en caso contrario, existe un control social que vigila al tramposo.

El segundo autor, Pinker (2012), demuestra que las curvas de violencia humana han ido disminuyendo, mientras aumentan otras positivas. Cada vez hay menos muertes violentas (como indica el autor, es una afirmación contraintuitiva ya que la gente cree que la sociedad va de mal en peor). Muchas conductas habituales y cotidianas de la antigüedad y de la edad media, como ir a ver ejecuciones públicas, quemar herejes o lapidar a infieles, hoy son impensables; al menos en la mayor parte del planeta. Por tanto, si se observa la historia, como hace el autor, se dibuja un ser humano violento y poco piadoso, en la que el perdón brillaba por su ausencia. Ahora esto ha cambiado.

Para Pinker la respuesta a cómo y por qué se ha llegado a un ser humano más positivo y civilizado viene del desarrollo y confluencia de diversas áreas: científico-técnicas y socio-políticas. Básicamente, hoy se vive con seguridad, necesidades básicas cubiertas, más salud, derechos y educación, entre otras mejoras. La psicología humana se adapta a estos tiempos, desarrollando o reprimiendo capacidades según los valores que precisa la cultura del momento. Así, se han fortalecido la empatía y la autonomía individual, frente a sentimientos negativos. Los nuevos valores se viven de forma natural. Natural significa que se insertan en la cultura como si siempre hubieran estado ahí.

En definitiva, hoy la gente desarrolla la empatía y la colaboración (y ensombrece otros sentimientos). Las ciencias recogen estos sentimientos y crean tendencias (que, sea como fuere, siguiendo a Wright, existirían con independencia del nombre de la corriente, los constructos o los autores; es decir, las buenas ideas podrían reprimirse y desaparecer, pero acabarían reapareciendo). Por ejemplo, el castigo que fue habitual y normal en el pasado es intolerable en el presente. Por tanto, la perspectiva ética, basada en valores socialmente aceptados, impone visiones y modelos que se deben seguir de una u otra forma.

La prueba de que los valores personales se sincronizan con la cultura reinante se observa cuando se estudian diferentes sociedades. Por ejemplo, la idea de igualdad entre géneros es obvia y natural en occidente, en cambio no se admite en ciertos lugares del mundo, quienes aceptan como natural la desigualdad. Esto 
demuestra que los derechos y los valores se logran tras un cambio, que no están ahí para recogerlos sin más y que la ciencia positiva precisa de ese cambio social. Henrich et al. (2010) se valen del concepto weird (que significa extraño, por un lado, y el acrónimo de occidental, educado, industrializado, rico y democrático, por otro) para subrayar que todas las conclusiones de la psicología (cabe incluir la positiva) provienen de occidente. En los artículos analizados por los autores, el $96 \%$ de los sujetos en base a los cuales se confeccionan las teorías eran ciudadanos weird (y, además, en su mayoría eran estudiantes universitarios). Por tanto, el triunfo de unos valores sobre otros no depende, solamente, de los conocimientos sino de la aceptación social.

De hecho, hay trabajos que demuestran las diferencias. En el de Bone et al. (2016) comparan a un grupo de ciudadanos weird (de USA) con uno no weird (de la India) en relación con los castigos que se aplican los jugadores cuando se defraudan en un experimento con teoría de juegos. En el occidente, weird se perdona más que en el oriente no weird. Tanto el perdón como el castigo son temas relevantes para la psicología positiva y el apoyo conductual positivo. Por su parte, Lyengar (2011) describe diferencias culturales con relación a elegir lo mejor para uno o lo mejor para todos. Así, según la autora, un $75 \%$ de estudiantes universitarios indios contraerían el matrimonio pactado por las familias, frente a un $14 \%$ de los estadounidenses. Por tanto, para los universitarios hindúes la felicidad se determina por el cumplimiento de los deberes familiares, pasando a un segundo plano la libre elección. Para un occidental esto es inadmisible. La autodeterminación es un concepto primordial para las personas con discapacidad. En definitiva, estos ejemplos señalan que la aplicación de los constructos (bienestar, calidad, perdón o autodeterminación) depende, en gran medida, del medio cultural.

\section{Conclusiones}

La psicología positiva y el apoyo conductual positivo se han especializado en la investigación y difusión del bienestar y la felicidad. Han despertado un indudable interés, revistas y asociaciones lo evidencian. Ambos modelos están hermanados en la positividad, por aplicar uno de los diversos términos que usan. Les diferencia su historia particular y las poblaciones a las que se dirigen. La psicología positiva a la población general aunque hoy incluye a las personas con discapacidad, excluidas anteriormente (Shogren et al., 2017; Wehmeyer, 2013b). También se ha comprobado que se está aplicando en escuelas ordinarias (Noble y McGrath, 2015; Seligman et al., 2009). Por su parte, el apoyo conductual positivo que se inició para personas con discapacidad y conductas problemáticas hoy va más allá y se ha generalizado a las escuelas ordinarias, juventud y tercera edad.

En este trabajo se ha indicado que las corrientes positivas pivotan entre distintos niveles, desde los conocimientos concretos (el lado empírico) hasta la difusión general de esos conocimientos (el modelo). En el debate de quienes lo defienden o lo critican se hallan, en ocasiones, argumentos que se mueven sin demasiada concreción por esos diferentes niveles. Esto dificulta el acuerdo y las conclusiones. Se podría pensar en sesgos debido a la denominada estrategia de contrastación positiva, un efecto psicológico que actúa para confirmar los juicios y las creencias. En definitiva, habría cierta relatividad en los argumentos. Por ejemplo, los críticos creen que el exceso de positividad puede acabar siendo negativo, lo que tal vez sea una obviedad que no nie- 
gan los defensores (si unos y otros están de acuerdo, luego qué discuten). Tal vez, se desconocen los auténticos detalles de este asunto. Es complejo determinar bajo qué circunstancia, cantidades, lugares y situaciones el binomio positivo-negativo cambia. Esto mismo se ha observado con el perdón en el matrimonio, que bajo unas circunstancias es positivo y bajo otras se vuelve negativo. En definitiva, los detalles y el impacto de los principios positivos son focos de interés para la investigación y deberán aportarse datos concretos que arrojen luz al respecto, más allá de las conclusiones genéricas y obvias por sentido común.

Para el apoyo conductual positivo se podría pensar que lo anterior es menos evidente, no obstante, también existe un cierto debate. Se ha comentado, por ejemplo, que al afrontar situaciones complejas que podrían solucionarse entregando un estímulo preferente sería, para algunos, una contradicción porque actuaría como un refuerzo; sin embargo, otros creen que las situaciones difíciles deben solucionarse en ese momento, aunque sea cediendo y reforzando (Spicer y Crates, 2016). Como quiera que sea, la positividad (hay que subrayarlo) tiene un matrimonio con la ética que condiciona las estrategias que se deben usar con independencia de la eficacia de los procedimientos.

También se ha comprobado que a los modelos positivos se les podría acusar de indefinición. Y es así, en cierta medida. Se ha descrito que la psicología positiva podría definirse de diez maneras diferentes. Piña (2015), con relación a la resiliencia, indica que puede significar una respuesta, un proceso o una cualidad personal. Para el apoyo conductual positivo esto sucede en menor medida porque se cobija bajo el paraguas del análisis conductual aplicado. De cualquier forma, entre las definiciones del apoyo conductual positivo también se observa disparidad. Por ejemplo, algunos introducen la calidad de vida explícitamente y otros no la incluyen, al menos expresamente. Como se ha descrito, los hay que definen el apoyo conductual positivo como análisis conductual aplicado más todos los conceptos actuales de los servicios. En definitiva, los constructos que utilizan los modelos positivos son amplios y de sentido común. Ahora bien, como indica Piña (2015), una vez que son parte de una teoría deben ser operativos e inequívocos, esto eliminaría confusión y sesgos. En último término, algunos interpretan que la flexibilidad de la definición es una fortaleza. Kincaid (2018) cree que el campo apoyo conductual positivo ha crecido y cambiado significativamente estos últimos 25 años y piensa que en el futuro está llamado a ser un campo abierto. Cabe la posibilidad, por tanto, de una definición abierta; no obstante, también cabe dibujar fronteras que definan aquellos conceptos que formarían parte de la misma. Son los modelos mismos quienes deberán explicarnos sus propios términos; por ahora el análisis conductual aplicado (las estrategias técnicas) y la calidad de vida parecen dos elementos bien posicionados en el apoyo conductual positivo.

Por otro lado, hoy la evolución de los conocimientos condiciona visiones eclécticas de la conducta humana. Es por eso que la psicología positiva que inició sus inquietudes sobre las variables personales, está abriéndose hacia los contextos. El apoyo conductual positivo, por su parte, desde sus inicios siempre ha considerado la función del ambiente porque durante los años 80 y 90 la filosofía de las intervenciones se basaba exclusivamente en los sujetos, es decir, se desarrollaban programas para modificar (solamente) las conductas sin considerar las condiciones de vida (Poplin, 1991). Posteriormente, debido al crecimiento de servicios y a los nuevos valores, los contextos ganaron peso. Así, el apoyo conductual positivo convirtió la modificación de conducta clásica en modificación de conducta de los ambientes. Por tanto, la descripción de las variables que condicionan la positividad considerando el binomio persona-contexto, y demás sinergias, es una de las tareas de la investigación en positividad. Por eso, las condiciones materiales y sociales (los contextos) deben describirse adecuadamente, sobre todo si hay contradicciones; es decir, si las condiciones de vida son muy complejas, duras o difíciles. Esto, para las personas con discapacidad que han 
vivido en entornos vulnerables se convierte en una necesidad incuestionable. Verdugo y Schalock (2006) lo han comentado: el progreso no ha producido cambios suficientes para las personas con discapacidad.

En cualquier caso, los modelos positivos se presentaban como una novedad, por eso, se ha introducido una reflexión que podría explicar el éxito y popularidad que han obtenido. Según esta reflexión, los modelos positivos trabajan en un campo previamente abonado. Se puede comprobar, por ejemplo, cuando se da formación a nuevos profesionales, que no tienen conocimientos previos en apoyo conductual positivo, y que presentan una sensibilidad y cultura positiva. Cuando a estos futuros profesionales se les narra las condiciones de vida de las instituciones donde vivían personas con discapacidad demuestran incomprensión y extrañeza. Por último, también se comprueba que los valores sobre bienestar están tan arraigados que se han expandido hasta niveles inimaginables anteriormente, por ejemplo, con el bienestar animal se demuestra una expansión más allá de lo humano. En definitiva, la explicación más económica es que la gente tiene el mismo lenguaje (valores y sentimientos) que el de los modelos positivos. Estos valores sociales son anteriores a la aparición de los modelos positivos. La investigación de los marcos culturales, con relación a la positividad, podría clarificar variables importantes para la formación y difusión del conocimiento.

Wrigth (2005), para comprender los marcos positivos, apela a la teoría de juegos. La dinámica descrita con esta teoría apela hacia una estructura invisible que motiva la aparición de nuevos valores. El concepto clave es el de equilibrio, es decir, la colaboración (cuando la dinámica posible es colaborar o defraudar). ¿Es relevante esta teoría optimista, tal como la plantea el autor, para el apoyo conductual positivo? La positividad, y mucho más en el apoyo conductual positivo, se basa en la colaboración y el apoyo social, por tanto, se trata de un equilibrio social (se comprende que colaborar en el bienestar del prójimo es colaborar en nuestro propio bienestar, aunque sea a futuro). Por otra parte, la observación del autor sobre la vigilancia que ejerce la gente, controlando continuamente la actuación ajena, condiciona para que el discurso positivo sea coherente, es decir, para que las posibles brechas entre la teoría y la práctica en positividad sean lo más convergentes posible.

Pinker (2012), por su parte, señala la tendencia social a acumular mejoras del mundo de las ideas e instituciones. Esta acumulación cambia las creencias y los valores. Los ejemplos son evidentes cuando se compara la crueldad del pasado con la sensibilidad actual. El autor subraya dos ideas relevantes. La primera, que el bienestar se asienta en otras mejoras como, por ejemplo, vivir en sociedades altamente seguras. La segunda explicaría el cambio psicológico como una adaptación cultural que presiona sobre sus miembros; por ejemplo, el desarrollo de una justicia garantista e institucionalizada inhibiría por innecesaria -y prohibida- la venganza. La empatía sería la lógica evolución de la convivencia organizada para logros comunes. Esta cultura se interioriza de forma natural, sobre todo para quienes no conocen otra. Esta visión de cultura como una sinergia puede ser una fuente de inspiración para la denominada cultura de los servicios. Así, esta visión señala la relación de los recursos (el contexto) con la cultura de forma contundente.

También es interesante señalar antecedentes concretos tanto de la psicología positiva como del apoyo conductual positivo. Por ejemplo, la psicología humanista, cuyos conceptos bien podrían pasar por psicología positiva: autorrealización, bondad natural, consideración positiva de las personas y aceptación incondicional. Para el apoyo conductual positivo, la enseñanza suave que también describía constructos positivos: solidaridad, vínculos o cuidado incondicional. Así pues, según como se presentan las teorías se podría estar invitando o inhibiendo la curiosidad hacía escuelas antecedentes.

En definitiva, la difusión de un modelo es un punto crítico, en tanto en cuanto los clínicos y el público general son quienes usan sus premisas. Hoy por hoy, los modelos están provocando aproximaciones, para 
la mayoría, y rechazos, para algunos. En este sentido, los modelos deben cuestionarse algunos aspectos: su lenguaje triunfalista y, tal vez, un lenguaje incuestionable y, a la vez, poco discriminativo. Al fin y al cabo, nadie está en contra del bienestar (ni los críticos). El deseo del bienestar para todos es una postura ética que debe difundirse, por tanto, no hay que menoscabar el papel de altavoz que tienen los modelos. Al mismo tiempo, sentirse desafiado por los críticos (a fin de analizar, aprender y cambiar si fuera preciso) parece una postura que beneficiaría (posiblemente está beneficiando) a la positividad.

Para finalizar, como se ha indicado, el apoyo conductual positivo trabaja en la provisión de servicios por lo que dirige el foco de la positividad hacia las personas y los ambientes en mayor medida que la psicología positiva. Como núcleo se ha resaltado la relación humana porque existen datos contundentes de su papel en el bienestar físico y psíquico. Por eso, las interacciones son un flujo continuo de intercambio concreto entre quienes proveen esos servicios, los profesionales o maestros y quienes lo reciben, los usuarios, alumnos o tutelados. Las relaciones humanas, frente a muchos de los amplios constructos usados, es una variable concreta y perceptible. Esto podría ser una aportación del apoyo conductual positivo a la -inevitable- positividad. 


\section{Referencias bibliográficas}

Berrocal, C. et al. (2008). Aplicaciones clínicas de la terapia del bienestar. En C. Vázquez y G. Hervás (Eds.), Psicología positiva aplicada (pp. 101-127). Desclée De Brouwer.

Bone, J. E. et al. (2016). Exploring the motivations for punishment: Framing and country-level effects. PLOS ONE, 11(8), e0159769. https://doi.org/10.1371/journal.pone.0159769.

Boothby, E. J. et al. (2017). The world looks better together: How close others enhance our visual experiences. Personal Relationships, 24(3), pp. 694-714. https://doi.org/10.1111/pere.12201.

van Boven, L. y Gilovich, T. (2003). To do or to have? That is the question. Journal of Personality and Social Psychology, 85(6), pp. 1193-1202. https://doi.org/10.1037/0022-3514.85.6.1193.

de la Cal, A. (2014). Psicología positiva en discapacidad intelectual. Programa para el desarrollo emocional y la fortaleza de gratitud. Universidad de Valladolid.

Carr, E. G. (2007). The expanding vision of positive behavior support: Research perspectives on happiness, helpfulness, hopefulness. Journal of Positive Behaviour Interventions, 9(1), pp. 3-14.

Carr, E. G. et al. (1996). Intervención comunicativa sobre los problemas de comportamiento. Alianza Psicología. (Trabajo original publicado en 1994).

Carr, E. G. et al. (1999). Positive behavior support for people with developmental disabilities. AAMR.

del Cerro, M. C. R. et al. (2010). Maternal care counteracts behavioral effects of prenatal environmental stress in female rats. Behavioural Brain Research, 208(2), pp. 593-602. https://doi.org/10.1016/j.bbr.2010.01.003.

Chaves, C. et al. (2016). A comparative study on the efficacy of a positive psychology intervention and a cognitive therapy for clinical depression. Cognitive Therapy and Research, 41, pp. 417-433. https://doi.org/10.1007/ s10608-016-9778-9.

Felce, D. y Perry, J. (1995). Quality of life: Its definition and measurement. Research in Developmental Disabilities, 16(1), pp. 51-74. https://doi.org/10.1016/0891-4222(94)00028-8.

Fernández-Abascal, E. G. (2009). Emociones positivas, psicología positiva y bienestar. En E. Fernández-Abascal (Ed.), Emociones positivas (pp. 27-46). Pirámide.

Fernández-Ríos, L. y Novo, M. (2012). Positive pychology: Zeigeist (or spirit of the times) or ignorance (or disinformation) of history? International Journal of Clinical and Health Psychology, 12(2), pp. 333-344.

Foxx, R. M. (2005). Severe agressive and self-destructive behavior: The myth of the nonaversive treatment of severe behavior. En J. W. Jacobson et al. (Eds.), Controversial therapies for developmental disabilities: Fad, fashion and science in professional practices (pp. 295-310). Lawrence Erlbaum Associates.

Han, B. C. (2016). La sociedad del cansancio. Herder (Trabajo original publicado en 2010).

Hassiotis, A. et al. (2018). Clinical outcomes of staff training in positive bahaviour support to reduce challenging behaviour in adults with intellectual disability: Cluster randomised controlled trial. The British Journal of Psychiatry, 212, pp. 161-168. https://doi.org/10.1192/bjp.2017.34.

Henrich, J. et al. (2010). The weirdest people in the world? Behavioral and brain sciences. 33(2-3), pp. 61-83. https://doi.org/10.1017/S0140525X0999152X. 
Holt-Lunstad, J. et al. (2010). Social relationships and mortality risk: A meta-analytic review. PLoS Medicine, 7(7), e1000316. https://doi.org/10.1371/journal.pmed.1000316.

Horner, R. H. et al. (1990). Toward a technology of «nonaversive» behavioral support. Journal of the Association for Persons with Severe Handicaps, 15(3), pp. 125-132.

Horner, R. H. y Sugai, G. (2018). Future directions for positive behavior support: A commentary. Journal of Positive Behavior Interventions, 20(1), pp. 19-22.

Johnston, J. M. et al. (2006). Positive behavior support and applied behavior analysis. The Behavior Analyst, 29(1), pp. 51-74. https://doi.org/10.1177/0145445508317132.

Jones, R. S. P. y McCaughey, R. E. (1992). Gentle teaching and applied behavior analysis: A critical review. Journal of Applied Behavior Analysis, 25(4), pp. 853-867. https://doi.org/10.1901/jaba.1992.25-853.

Kincaid, D. (2018). Staying true to our PBS roots in a changing world. Journal of Positive Behavior Interventions, 20(1), pp. 15-18. https://doi.org/10.1177/1098300717735057.

Lavigna, G. W. et al. (2009). Positive practices in behavioral support. Through non-linear applied behavior analysis (facilitator's manual). IABA.

Linley, P. A. et al. (2006). Positive psychology: Past, present, and (possible) future. The Journal of Positive Psychology, 1(1), pp. 3-16. https://doi.org/10.1080/17439760500372796.

Lupano, M. L. y Castro, A. (2010). Psicologia positiva: un análisis desde su surgimiento. Ciencias Psicológicas, 4(1), pp. 45-56.

Lyengar, S. (2011). El arte de elegir. Gestión 2000 (Trabajo original publicado en 2010).

LyubomirsKy, S. et al. (2005). The benefits of frequent positive affect: Does happiness lead to success? Psychological Bulletin, 131(6), pp. 803-855.

Manokara, V. et al. (2018). Exploring the knowledge and competency of positive behavioural support (PBS) amongst disability professionals in Singapore. International Journal of Positive Behavioural Support, 7(2), pp. 49-58.

McNulty, J. K. y Fincham, F. D. (2012). Beyond positive psychology? Toward a contextual view of psychological processes and well-being. American Psychologist, 67(2), pp. 101-110. https://doi.org/10.1037/a0024572.

Mulick, J. A. y Butter, E. M. (2005). Positive behavior support: A paternalistic utopian delusion. En J. W. Jacobson et al. (Eds.), Controversial therapies for developmental disabilities: Fad, fashion and science in professional practices (pp. 385-404). Lawrence Erlbaum Associates.

Newson, C. y Kroeger, K. (2005). Nonaversive treatment. En J. W. Jacobson et al. (Eds.), Controversial therapies for developmental disabilities: Fad, fashion and science in professional practices (pp. 405-422). Lawrence Erlbaum Associates.

Noble, T. y McGrath, H. (2015). Prosper: A new framework for positive education. Psychology of Well-Being, 5(2). https://doi.org/10.1186/s13612-015-0030-2.

Park, N. et al. (2013). La psicología positiva: investigación y aplicaciones. Terapia Psicológica, 31(1), pp. 11-19.

Pérez-Álvarez, M. (2012). La psicología positiva: magia simpática. Papeles del Psicólogo, 33(3), pp. $183-201$. http://www.papelesdelpsicologo.es/pdf/2137.pdf.

Pérez-Álvarez, M. (2013). La psicología positiva y sus amigos: en evidencia. Papeles del Psicólogo, 34(3), pp. 208-226. 
Pérez-Álvarez, M. et al. (2018). La vida real en tiempos de la felicidad. Crítica de la psicología (y de la ideología) positiva. Alianza Editorial.

Piña, J. A. (2014). La psicología positiva ¿Ciencia y práctica de la psicología? Papeles del Psicólogo, 35(2), pp. 144-158.

Piña, J. A. (2015). Un análisis crítico del concepto de resiliencia en psicología. Anales de Psicología, 31(3), pp. 751-758. https://doi.org/10.6018/analesps.31.3.185631.

Pinkelman, S. E. y Horner, R. H. (2019). Applying lessons from the teaching-family model: Positive behavioral interventions and supports (PBIS). Perspectives on Behavior Science. 42(2), pp. 233-240. https://doi.org/10.1007/ s40614-019-00199-x.

Pinker, S. (2012). Los ángeles que llevamos dentro. El declive de la violencia y sus consecuencias. Paidós (Trabajo original publicado en 2011).

Poplin, M. S. (1991). La falacia reduccionista en las discapacidades para el aprendizaje: duplicación del pasado por reducción del presente. Siglo Cero, 137, pp. 18-28. https://sid.usal.es/articulos/discapacidad/4191/8-2-6/ la-falacia-reduccionista-en-las-discapacidades-para-el-aprendizaje-duplicacion-del-pasado-por-reducciondel-presente.aspx.

Rifkin, J. (2010). La civilización empática. Paidós (Trabajo original publicado en 2009).

Schalock, R. L. y Verdugo, M. A. (2006). Revisión actualizada del concepto de calidad de vida. En M. A. Verdugo (Ed.), Cómo mejorar la calidad de vida de las personas con discapacidad. Instrumentos y estrategias de evaluación (pp. 29-41). Amaru Editores.

Seligman, M. E. P. et al. (2009). Positive education: Positive psychology and classroom interventions. Oxford Review of Education, 35(3), pp. 293-311. https://doi.org/10.1080/03054980902934563.

Shogren, K. A. et al. (Eds.) (2017). Handbook of positive psychology in intellectual and developmental disabilities. Translating research into practice. Springer.

Spicer, M. y Crates, N. (2016). Non-aversive reactive strategies (NARS) o reduce episodic severity of aggression and to reduce the need for restrictive practices. En R. P. Liberman y G. W. La Vigna (Eds.), New directions in the treatment of aggressive behavior (pp. 323-360). Nova Science Publishers.

Taleb, N. (2016). Antifrágil: las cosas que se benefician del desorden. Booket (Trabajo original publicado en 2012).

Vázquez, C. (2013). La psicología positiva y sus enemigos: una réplica en base a la evidencia científica. Papeles del Psicólogo, 34(2), pp. 91-115. https://www.redalyc.org/pdf/778/77827025002.pdf.

Vázquez, C. y Hervás, G. (2008). Salud mental positiva: del síntoma al bienestar. En C. Vázquez y G. Hervás (Eds.), Psicología positiva aplicada (pp. 17-39). Desclée de Brouwer.

Vázquez, C. y Hervás, G. (2009). La ciencia del bienestar. En C. Vázquez y G. Hervás (Eds.), La ciencia del bienestar: fundamentos de una psicología positiva (pp. 13-46). Alianza.

Vera, B. (2006). La psicología positiva: una nueva forma de entender la psicología. Papeles del Psicólogo, 27(1), pp. 3-8.

Verdugo, M. A. y Schalock, R. L. (2006). Aspectos claves para medir la calidad de vida. En M. A. Verdugo (Ed.), Cómo mejorar la calidad de vida de las personas con discapacidad. Instrumentos y estrategias de evaluación (pp. 43-58). Amarú Ediciones. 
Wehmeyer, M. L. (Ed.). (2013a). Beyond pathology: Positive psychology and disability. En M. L. Wehmeyer (Ed.), The Oxford handbook of positive psychology and disability (pp. 3-6). Oxford University Press.

Wehmeyer, M. L. (Ed.) (2013b). The Oxford handbook of positive psychology and disability. Oxford University Press.

Wrigth, R. (2005). Nadie pierde: la teoría de juegos y la lógica del destino humano. Tusquets (Trabajo original publicado en 1999).

Yakushko, O. y Blodgett, E. (2018). Negative reflections about positive psychology: On constraining the field to a focus on happiness and personal achievement. Journal of Humanistic Psychology, 61(1), pp. 104-131. https:// doi.org/10.1177/0022167818794551. 